\title{
Calcium triggers exit from meiosis II by targeting the APC/C inhibitor XErp1 for degradation
}

\author{
Nadine R. Rauh ${ }^{1 *}$, Andreas Schmidt ${ }^{1 *}$, Jenny Bormann' ${ }^{1}$ Erich A. Nigg ${ }^{2} \&$ Thomas U. Mayer ${ }^{1}$
}

Vertebrate eggs awaiting fertilization are arrested at metaphase of meiosis II by a biochemical activity termed cytostatic factor $(\mathrm{CSF})^{1,2}$. This activity inhibits the anaphase-promoting complex/ cyclosome (APC/C), a ubiquitin ligase that triggers anaphase onset and mitotic/meiotic exit by targeting securin and M-phase cyclins for destruction ${ }^{3,4,5}$. On fertilization a transient rise in free intracellular calcium ${ }^{6}$ causes release from CSF arrest and thus APC/C activation. Although it has previously been shown that calcium induces the release of $\mathrm{APC} / \mathrm{C}$ from $\mathrm{CSF}$ inhibition through calmodulin-dependent protein kinase II (CaMKII) ${ }^{7,8}$, the relevant substrates of this kinase have not been identified. Recently, we characterized XErpl (Emi2), an inhibitor of the APC/C and key component of CSF activity in Xenopus egg extract ${ }^{9}$. Here we show that calcium-activated CaMKII triggers exit from meiosis II by sensitizing the APC/C inhibitor XErp1 for polo-like kinase 1 (Plx1)-dependent degradation. Phosphorylation of XErpl by CaMKII leads to the recruitment of Plxl that in turn triggers the destruction of XErp1 by phosphorylating a site known to serve as a phosphorylation-dependent degradation signal. These results provide a molecular explanation for how the fertilization-induced calcium increase triggers exit from meiosis II.
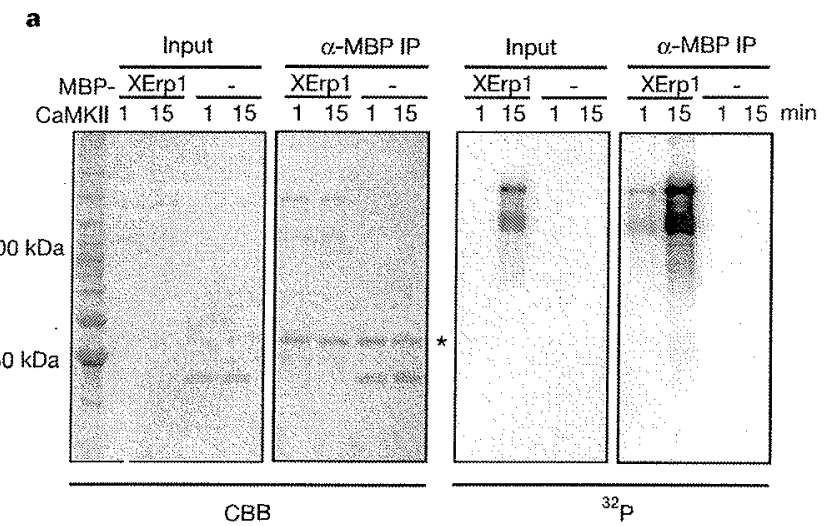

c

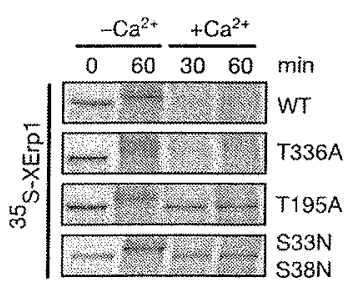

d

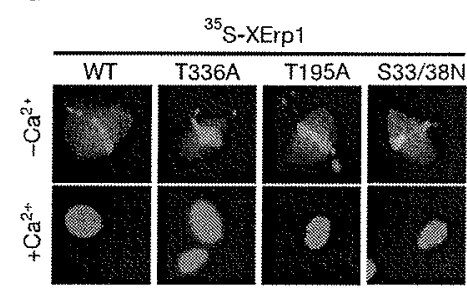

Although it is well established that CaMKII is the essential target of the calcium signal ${ }^{7,8}$ on fertilization, the relevant substrates of this kinase have not been identified, and the mechanism(s) leading to $\mathrm{APC} / \mathrm{C}$ activation have long remained obscure. Recently, we have identified XErp1, a novel component of CSF activity that is both necessary and sufficient to keep the APC/C inactive in CSF-arrested Xenopus egg extracts ${ }^{9}$ (CSF extracts). In response to calcium, XErpI is rapidly degraded via a $\mathrm{Plxl}$-dependent mechanism, leading to CSF release and $\mathrm{APC} / \mathrm{C}$ activation. We have further shown that XErp1 destruction depends on phosphorylation of two critical serine residues within a motif (DSGX ${ }_{3} S$ ) known to serve as a 'phosphodegron' for the ubiquitin ligase complex Skpl-Cullin-F-box ${ }^{\beta-\Gamma R C P}$ $\left(\mathrm{SCF}^{\beta-T R C P} ;\right.$ ref. 10$)$. Although the identification of XErpl as a critical substrate of $\mathrm{Plx} 1$ provided an attractive explanation for the essential function of PlxI in APC/C activation ", the role of the calcium signal remained unclear. In particular, it remained to be explained why XErp1 is not targeted for degradation in CSF extracts, despite the presence of active Plx1 and SCF ${ }^{\beta-T R C P}$. Here we have explored the hypothesis that a calcium-dependent mechanism could sensitize XErpl for phosphorylation by Plxl.

We first asked whether XErp1 could be a substrate of CaMKII.
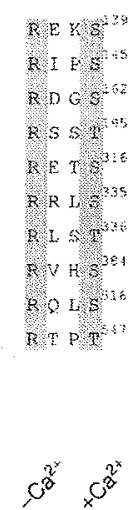

e.

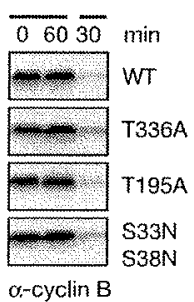

Figure 1 | XErp1 is a substrate of CaMKII in vitro. a, MBP-tagged XErp ${ }^{\text {WT }}$ or MBP alone were subjected to CaMKII phosphorylation reactions for the indicated times and immunopurified using antiMBP antibodies (asterisk marks heavy chains). $\mathrm{CBB}$ indicates Commassie Brilliant Blue. The incorporation of ${ }^{32} \mathrm{P}$ was analysed by autoradiography. b, Amino acid sequence of all putative CaMKII phosphorylation sites (RXXS/T) present in XErp1, c e, Both DSGX $3 S^{38}$ and RXST $^{195}$ motifs are essential for the degradation of XErpl on calcium-stimulation. ${ }^{35} \mathrm{~S}$-labelled IVT XErpl proteins were incubated in CSF extract in the presence or absence of calcium. At the indicated time points samples were withdrawn for analysis by autoradiography (c), microscopic examination of chromatin and spindle structures (d) and immunoblotting for cyclin B (e).

Chemical Biology, Independent Research Group, ${ }^{2}$ Department of Cell Biology, Max Planck Institute of Biochemistry, Am Klopferspitz 18, D-82152 Martinsried, Germany. "These authors contributed equally to this work. 
Autoradiographic analyses revealed that CaMKII efficiently phosphorylated purified maltose-binding protein (MBP)-tagged wildtype XErp1 but not MBP itself in vitro (Fig. la). Together with our previous results that XErpl degradation depends on its prior phosphorylation by Plxl (ref. 9), this result raised the question of how CaMKII and Plxl cooperate in response to a calcium signal to target XErp1 for destruction. One attractive model was suggested by recent findings implicating the noncatalytic carboxy terminus of Plks, the so-called polo-box domain (PBD), in both Plk activation and the targeting of these kinases to serine- or threonine-phosphorylated substrates ${ }^{12}$. Based on this concept it was tempting to speculate that CaMKII sensitizes XErpl for degradation by creating a binding site for Plx1. To explore this hypothesis, we examined the XErp1 sequence for sites that could potentially serve as CaMKII-regulated PBD binding sites (RXST/S; ref. 13). Of the ten putative CaMKII phosphorylation sites (RXXS/T) (Fig. 1b) only two sites (RXST ${ }^{195}$ and RXST $^{336}$ ) match this optimal consensus. To identify the CaMKII site(s) relevant for calcium-regulated degradation of XErp1 we examined the stability of in vitro translated (IVT), ${ }^{35} \mathrm{~S}$-labelled XErpl mutated at Thr 195 (XErp1 ${ }^{\text {T195A }}$ ) or Thr 336 (XErp $1^{\text {T336A }}$ ) in calcium-supplemented CSF extract. As shown previously ${ }^{4}$ IVT wild-type XErp ${ }^{W T}$ was rapidly degraded on anaphase onset whereas XErp1 mutated at its $\operatorname{DSGX}_{3} S$ motif $\left(X_{E r p 1}{ }^{\text {S3,3N,S38N }}\right.$ ) was not targeted for calcium-induced degradation (Fig. 1c). Notably, IVT XErp $1^{\mathrm{T} 195 \mathrm{~A}}$, but not XErp $1^{\mathrm{T} 336 \mathrm{~A}}$, remained stable on calcium addition (Fig. 1c), indicating that Thr 195, but not Thr 336, is essential for the cell-cycle-regulated degradation of XErpI. As shown by microscopic analyses and by immunoblotting for cyclin $B$, all extracts had entered interphase on calcium addition (Fig. 1d, e), confirming that the trace amounts of IVT products added did not block calcium-induced CSF release. Consistent with our previous observations $^{4}$ higher concentrations of XErp $1^{\text {WT }}$ and XErp1 ${ }^{\mathrm{T}[\omega 5 \mathrm{~A}}$ were capable of blocking calcium-induced CSF release (see Supplementary Fig. S1). Taken together, these data are consistent with the model that CaMKII and Plxl cooperate to target XErpl for degradation on a calcium stimulus and that Thr 195 and the DSGX $\mathrm{S}_{3} \mathrm{~S}$ motif are both required for the cell-cycle-regulated destruction of XErp1.

Previous studies have shown that phosphopeptide binding to the Plk-PBD is favoured by a serine residue in position -1 (SpT/pS; ref. 13). The above model thus predicted that Ser 194 might contribute to sensitizing XErp1 for Plxl-dependent degradation. Indeed, ${ }^{35} \mathrm{~S}$ labelled IVT XErp ${ }^{\mathrm{S} 194 \hat{A}}$ remained stable in calcium-supplemented CSF extract (Fig. 2a, b), similar to XErp $1^{\mathrm{T} 195 \mathrm{~A}}$ and XErp1 ${ }^{\mathrm{S} 33 \mathrm{~N}, \mathrm{~S} 38 \mathrm{~N}}$. To prove that Plx1-binding to XErpl depends on both Ser 194 and Thr 195, MBP-tagged wild-type protein and appropriate mutants were subjected to far-western experiments. Indeed, phosphorylation of XErp ${ }^{\text {WI }}$ by CaMKII strongly enhanced the interaction between XErpl and purified full-length Plx1. As expected, wild-type PBD $\left(\mathrm{PBD}^{\mathrm{WT}}\right)$ but not a mutant form of $\mathrm{PBD}\left(\mathrm{PBD}^{\text {mut }}\right)$ was capable of binding to phosphorylated XErp 1 WT (Fig. 2c). In contrast, both the S194A and T195A mutants of XErpl treated with CaMKII failed to interact significantly with full-length Plx1 or PBD ${ }^{\mathrm{WT}}$ (Fig. 2c), indicating that RXST ${ }^{195}$ constitutes a CaMKII-regulated PlxI binding site. Mutation of the DSGX 3 S motif did not interfere with the ability of XErpI to bind to Plxl or the PBD ${ }^{\text {Wr }}$ on phosphorylation by CaMKII (Fig. 2c), in line with the expected order of events. Taken together, these data strongly suggest that CaMKII phosphorylation of XErpl on Thr 195 creates a binding site for Plx1, consistent with the idea that CaMKII sensitizes XErpl for Plx1-dependent degradation on anaphase onset.

An additional corollary of the proposed model is that PBD docking to XErp1 stimulates the kinase activity of Plx1 towards its substrate XErpl. To test this prediction, we used MBP-XErpI with or without prior phosphorylation (with unlabelled ATP) by CaMKII to carry out Plxl kinase assays in the presence of ${ }^{32}$ P-labelled ATP. Compared to untreated XErpI ${ }^{\text {WT }}$, XErp $1^{\text {Wr }}$ pre-phosphorylated by

CaMKII represented a much better in vitro substrate for PIx1 (Fig. 2d) consistent with enhanced Plxl recruitment after CaMKII phosphorylation. To rule out a contribution of CaMKII to the incorporation of ${ }^{32} \mathrm{P}$ into XErpl, parallel phosphorylation experiments were performed in the absence of Plxl. As shown in Fig. $2 \mathrm{~d}$, no labelling of XErpl with ${ }^{32} \mathrm{P}$ was observed under these conditions. Notably, pretreatment of MBP-XErpl ${ }^{\mathrm{S1} 194 \mathrm{~A}}$ or -XErp ${ }^{\mathrm{T} 195 \mathrm{~A}}$ did not result in increased phosphorylation by Plx 1 (Fig. 2d), confirming the inability of these mutants to provide a docking site for Plx1. Taken together, these data demonstrate that calcium-activated CaMKII converts XErpl into an efficient Plxl substrate.

We next wanted to confirm that the activity of CaMKII is essential for the degradation of XErp1 on anaphase onset. However, as the inhibition of CaMKII prevents CSF release, and thus XErpl degradation, we analysed the stability of IVT XErp1 in extract that was arrested at anaphase by the presence of non-degradable cyclin $B$ (refs 14,15 ). These extracts, called ' $\Delta 90$ extracts' (refs 14,15 ), retain

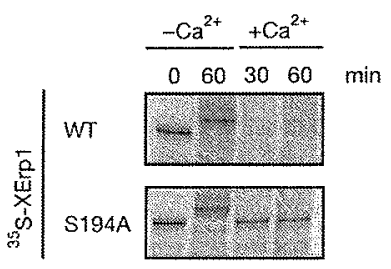

c
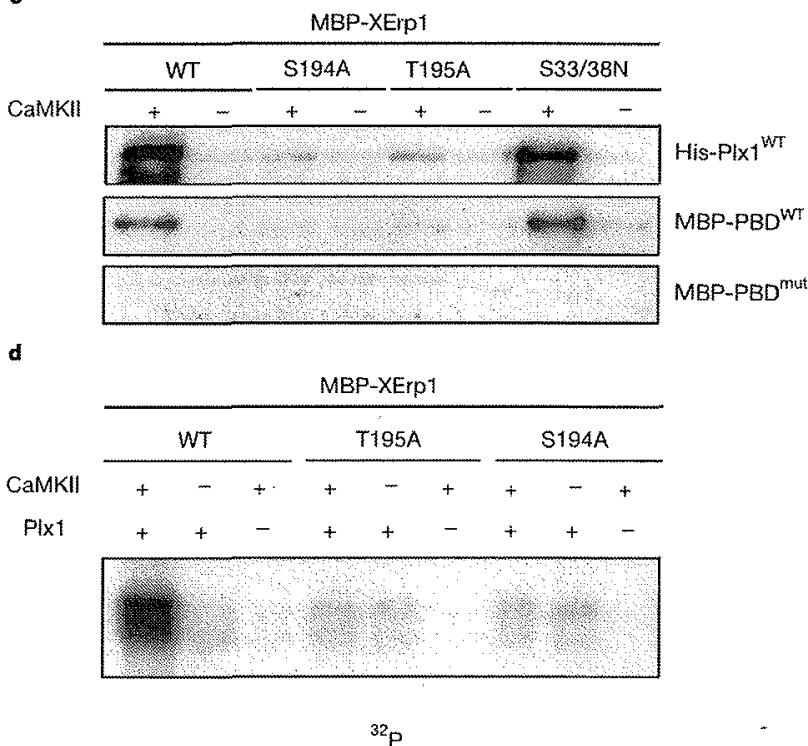

Figure 2 | CaMKII converts XErp1 into an efficient PIx1 substrate. a, b, Ser 194 is critical for the calcium-induced degradation of XErpl, IVT ${ }^{35} \mathrm{~S}$-XErp $1{ }^{\mathrm{WT}}$ or -XErp ${ }^{\mathrm{S194A}}$ was incubated in CSF extract in the presence or absence of calcium and samples were analysed by autoradiography (a) and microscopic examination of spindle and DNA morphology (b).

c, Phosphorylation of XErpl by CaMKII strongly enhances binding of Plx to XErpl. The binding of His-Plx1, MBP-PBD ${ }^{14 \mathrm{~T}}$ and MBP-PBD ${ }^{\mathrm{mut}}$ to MBPXErpI was analysed by far-western ligand blots. MBP-XErpl proteins were subjected to CaMKII phosphorylation reactions or mock treatments and resolved by SDS-PAGE. Bound PlxI and PBD were detected using purified anti-Plx1 antibodies. d, MBP.XErpl was subjected to in vitro phosphorylation reactions using recombinant PIxI. The incorporation of ${ }^{32} \mathrm{P}$ was analysed by autoradiography. As indicated, MBP-XErpl was preincubated with activated CaMKII (or subjected to a mock kinase reaction) and unlabelled ATP. To control for incorporation of ${ }^{32} \mathrm{P}$ by CaMKII, paralle reactions were performed in the absence of Plxl. 
CaMKII activity (see Supplementary Fig. S2) which can be modulated without affecting cell cycle progression. As expected, IVT $\mathrm{XErp}^{\mathrm{WT}}$ was rapidly degraded in $\Delta 90$ extracts (see Supplementary Fig. S2). As most available specific CaMKII inhibitors are not able to inhibit activated CaMKII but only to prevent CaMKII activation we first suppressed CaMKII activity in $\Delta 90$ extracts by the addition of EGTA (see Supplementary Fig. S2), allowing us to study the effect of a specific CaMKII inhibitor on the stability of XErp1. Consistently, in the absence of an additional calcium stimulus IVT XErp $1^{\text {WT }}$ remained stable in EGTA-treated $\Delta 90$ extracts, whereas it was rapidly degraded on calcium addition in a Thr 195-dependent manner (Fig. 3a). Notably, the calcium-induced degradation of XErp1 $1^{\text {WT }}$ could be significantly suppressed by the addition of $300 \mu \mathrm{M}$ CaM$\mathrm{KII}^{281-309}$ (Fig. 3a), a peptide known to specifically inhibit the calcium-induced activation of CaMKII (ref. 16). CaMKII activity assays demonstrated that CaMKII ${ }^{2.81-309}$ prevented the calciuminduced reactivation of CaMKII (Fig. 3c). The rapid degradation of IVT securin (Fig. 3a) under all conditions confirmed that the different treatments did not interfere with anaphase arrest. Furthermore, addition of a constitutively active form of CaMKII $\left(\right.$ CaMKII ${ }^{1-290}$ ) induced the rapid degradation of IVT XErp1 ${ }^{\text {WT }}$ but not of XErp1 ${ }^{\text {Tros }}$ (Fig. 3b) confirming that CaMKII is the kinase triggering XErpl degradation on calcium stimulus. In view of an ongoing debate about a possible contribution of Emil to CSF activity $^{17}$, we also used the above system to examine the fate of this XErp1-related protein. We found that CaMKII activity is not essential for the degradation of Emil, as indicated by the fact that IVT Emil was efficiently degraded in EGTA-treated $\Delta 90$ extracts even without calcium addition (Fig. $3 \mathrm{a}$ ), or when the calcium-induced activation of CaMKII was suppressed by the addition of CaMKII ${ }^{281-309}$ (Fig. 3a). Taken together, these data suggest that XErp1, but not Emil, is the critical target of calcium-activated CaMKII. This conclusion is in line with results showing that Emil is highly unstable in CSF-arrested extract ${ }^{18.19}$, from which CaMKII activity is naturally absent. The observation that Emil stability does not seem to be regulated by CaMKII, together with results showing that Emil protein is unstable in CSF-arrested egg extract ${ }^{18,19}$, argues against a critical contribution of Emil to CSF activity in Xenopus eggs.

The above data suggested that the lack of CaMKII activity accounts for the stability of XErp1 in CSF-arrested Xenopus egg extract. Consequently, we reasoned that a mutant XErp1 capable of serving as an efficient Plxl substrate independently of CaMKII should be targeted for degradation in CSF extract even in the absence of a calcium signal. To test this idea, we converted the CaMKII site (RXST ${ }^{195} \mathrm{LXD}$ ) into a consensus site for cyclin dependent kinase 1 (Cdk1; RXST ${ }^{195}$ PXK) and examined the stability of the ${ }^{35} \mathrm{~S}$-labelled

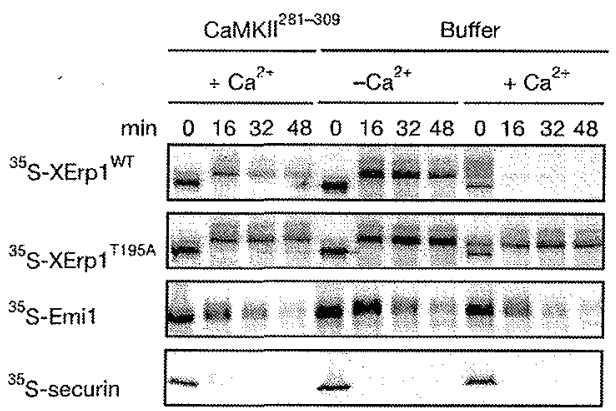

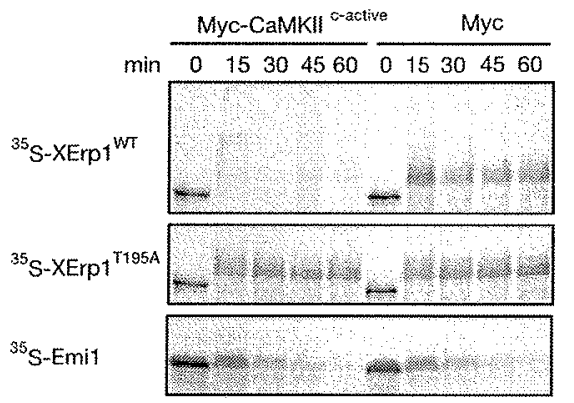

d

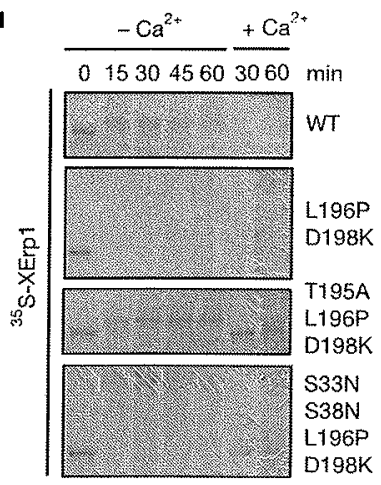

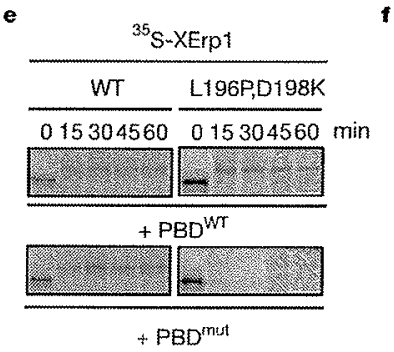

Figure 3 | CaMKII activity is essential for the degradation of XErp1 in Xenopus egg extract. a, IVT ${ }^{35} \mathrm{~S}-\mathrm{XErp} \mathrm{I}^{\text {WT }},-\mathrm{XErp}^{\mathrm{T}}{ }^{195 \mathrm{~A}}$, -Emil or -securin was incubated in $\Delta 90$ extract supplemented with buffer, or calcium in the presence or absence of $300 \mu \mathrm{M}$ of the CaMKII inhibitory peptide CaMKII ${ }^{281-309}$. Samples were analysed by autoradiography. b, IVT ${ }^{35}$ S-XErp $1{ }^{\text {WT }}$, -XErp $1^{\text {T195A }}$ or -Emil was incubated in $\Delta 90$ extract supplemented with immunopurified IVT constitutively active CaMKII ${ }^{\text {cactive }}$ or immunopurified mock IVT and analysed as in a. c, Samples from extracts shown in a were analysed for CaMKII activity. $d$, CdkI can take on CaMKII's function in sensitizing XErpl for Plxl-dependent degradation. IVT

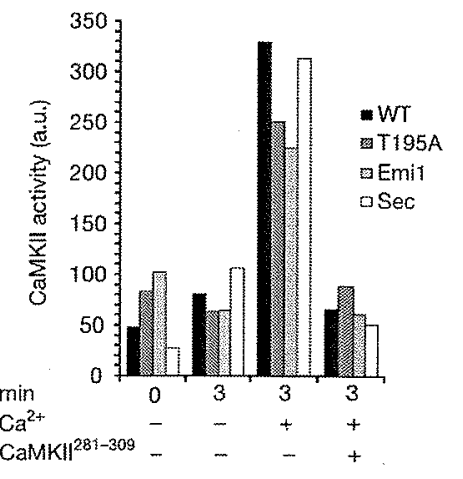

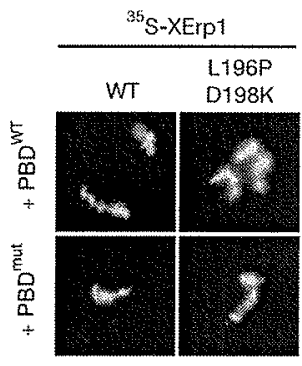

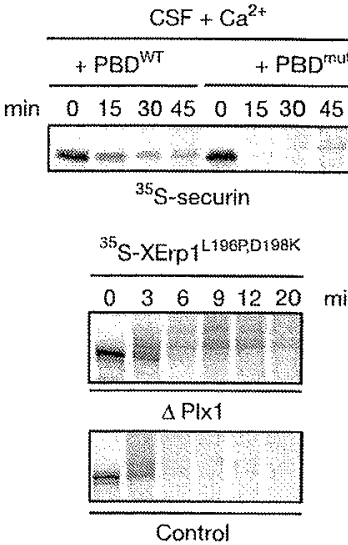

Control

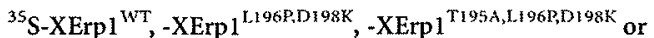
-XErp $1^{\text {S33N,S38N.196POI98K }}$ was incubated in CSF extract and samples were analysed by autoradiography. e-h Plxl activity is required for the degradation of XErp $1^{\text {E } 96 \%, D 19 B K}$ in CSF extract. e, $f$, Samples of CSF extract supplemented with MBP-PBD ${ }^{\text {WT }}$ or MBP-PBD ${ }^{\text {mut }}$ were analysed (e) as in d, and assayed for DNA morphology (f). CSF extracts treated as in e were supplemented with calcium and assayed for the stability of IVT securin (g). PlxI-depleted $(\triangle \mathrm{Plx} 1)$ or mock depleted (control) CSF extract were treated as in $\mathbf{d}(\mathbf{h})$. 
IVT product in CSF extracts. As Cdk1 is active in CSF extract and able to create docking sites for Plk-PBDs (ref. 13), the prediction was that Cdk1-mediated phosphorylation of this mutant XErpl would result in the docking of Plx1, the phosphorylation of the amino-terminal phospho-degron and the destruction of the protein. Indeed, whereas IVT XErpl ${ }^{\text {WT }}$ remained stable in CSF extract, XErpl ${ }^{\mathrm{L} 196 \mathrm{P}, \mathrm{D} 198 \mathrm{~K}}$ was rapidly degraded even in the absence of calcium (Fig. 3d), indicating that Cdk1 or any other proline-directed kinase active in CSF extract could substitute for CaMKII and sensitize this XErp1 mutant for Plxl-dependent degradation. As predicted by our model, the degradation of these XErp1 forms in CSF extract could be prevented by mutating either critical residues within the CDK1 consensus site (XErp1 ${ }^{\text {T195A,L196P.DI98K) }}$ or the DSGX $3 \mathrm{~S}^{38}$ motif

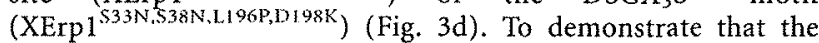
activity of Plx1 was essential for the degradation of XErp1 $1^{\text {L. 196P,D198K, }}$ we examined the stability of IVT XErp1 $1^{\text {I.1961 DD D198K }}$ in CSF extract supplemented with an excess of MBP-PBD ${ }^{\text {WT }}$, which we have shown to exert a dominant-negative effect on $\mathrm{Plxl}$ function ${ }^{3}$. As expected, the addition of MBP-PBD ${ }^{\text {WT }}$ resulted in a significant stabilization of IVT XErp $1^{\mathrm{L} 196 \mathrm{P}, \mathrm{DP} 198 \mathrm{~K}}$ in CSF extract, as compared to a controltreated extract (Fig. 3e) but had no effect on the stability of IVT XErp ${ }^{\text {WT }}$ (Fig. 3e). Analyses of the chromatin structures revealed that the extracts remained CSF-arrested under all conditions (Fig. 3f). The dominant-negative effect of MBP-PBD on Plxl function was confirmed in parallel experiments in which we examined the stability of IVT securin on calcium addition. PBD ${ }^{W T}$ - supplemented CSF extract, but not $\mathrm{PBD}^{\text {mut }}$-treated extract, failed to exit meiosis on calcium treatment, as indicated by stable IVT securin (Fig. $3 \mathrm{~g}$ ). Consistently, MBP-PBD ${ }^{\text {WT }}$ but not PBD ${ }^{\text {matit }}$ prevented the calciuminduced degradation of endogenous XErpl (see Supplementary Fig. S3). Finally, a similar stabilization of IVT XErp1 $1^{\text {L96 P.DD198K }}$ was observed in CSF extract immunodepleted of Plxl but not in mockdepleted extract (Fig. 3h). Taken together, these data demonstrate that the creation of a PlxI-PBD docking site on XErpl, an event normally brought about by calcium-activated CaMKII, defines the timing of Plxl-dependent degradation of XErpl.

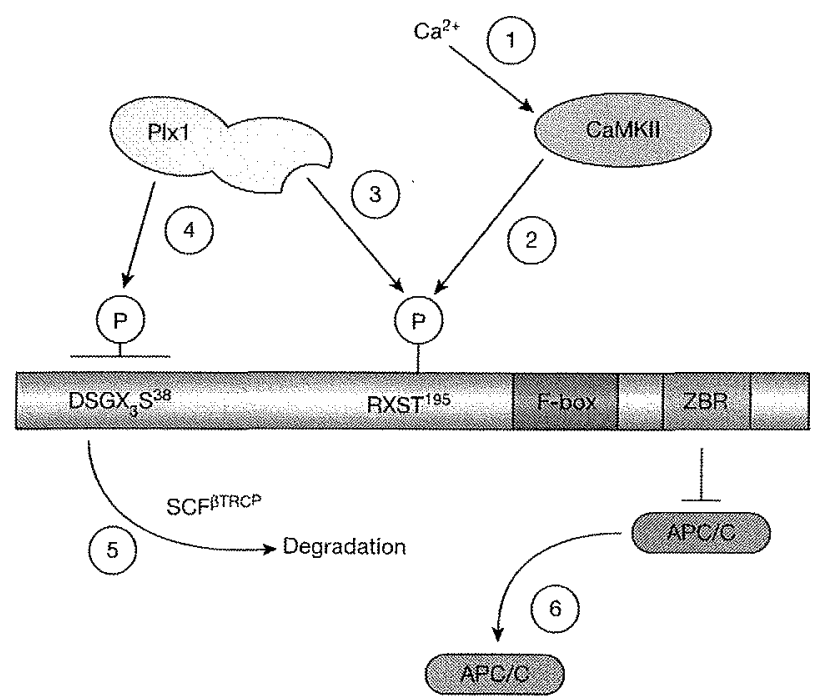

Figure 4 | Model of how calcium triggers release from CSF arrest. (1) A transient rise in free calcium activates CaMKII. (2) Activated CaMKII phosphorylates XErpl at Thr 195, thereby creating a docking site for Plx1. (3) Plxl binds to XErpl via its PBD. (4) On binding to its substrate, Plxl becomes activated and phosphorylates XErpl at Ser $33 / 38$ of the DSGX $\mathrm{D}_{3}^{38}$ motif. (5) Phosphorylated DSGX $S^{38}$ is recognized by $S C F^{\beta-T R C P}$ leading to the destruction of XErpl. (6) XErpl degradation leads to APC/C activation.
In conclusion, our study identifies the critical substrate of the calcium-activated CaMKII that triggers exit from meiosis II in response to fertilization. Specifically, our data demonstrate that CaMKII phosphorylates the $\mathrm{APC} / \mathrm{C}$ inhibitor $\mathrm{XErpl}$, thereby acting as a novel priming kinase for the recruitment of Plxl (Fig. 4). The bound Plx1, previously shown to be essential for causing release from CSF arrest", then triggers the degradation of XErpl by phosphorylation of a $\mathrm{DSGX}_{3} S$ degron. Our findings thus explain how a calcium signal prompts the exit from meiosis through the spatiotemporal integration of the action of two key kinases, CaMKII and Plx1, both converging onto XErpl, a critical inhibitor of APC/C.

\section{METHODS}

Plasmids, proteins and antibodies. XErp 1, Emil and securin constructs were as described ${ }^{4}$. Site-directed mutagenesis was performed using the QuikChange kit (Stratagene). MBP-tagged proteins were purified following published protocols $s^{2}$. Purification of His-tagged Plxl from SF9 cells was performed as described". In-vitro translation experiments using ${ }^{35} \mathrm{~S}$-labelled methionine were performed according to manufacturer's protocol (Promega). Full-length Plxl purified from SF-9 cells was used to generate rabbit antibodies to Plxl. MBP-PBD $^{\mathrm{WT}}$ and MBP-PBD ${ }^{\text {mut }}$ (W408F, H532A and K534A) were used as described previously".

In vitro kinase assays. CaMKII was purchased from New England biolabs. In vitro CaMKII assays were performed at $30^{\circ} \mathrm{C}$ in kinase reaction buffer containing $45 \mathrm{U}$ of activated CaMKII, $0.1-1 \mathrm{mM}$ ATP, $4 \mu \mathrm{Ci}\left[\gamma^{32} \mathrm{P}\right] \mathrm{ATP}$ and $200 \mathrm{ng}$ of purified MBP-tagged XErpl protein. Samples were taken at the indicated time points. CaMKII activity assays were performed based on published protocols"s using Autocamtide-2 (New England Biolabs) as substrate. Plxl kinase assays were performed for $5 \mathrm{~min}$ at $30^{\circ} \mathrm{C}$ using kinase reaction buffer supplemented with $4 \mu \mathrm{Ci}\left[\gamma^{32} \mathrm{P}\right] \mathrm{ATP}$ and MBP-tagged XErpl proteins which had previously been incubated with CaMKII or buffer (control) in the presence of 1 mM ATP. Far-western ligand blots. Far-western ligand blots were performed in TBS supplemented with $0.1 \%$ Tween- 20 and $5 \% \mathrm{wt} / \mathrm{vol}$ skim milk powder. For each assay $500 \mathrm{ng} \mathrm{MBP}-\mathrm{XErpl}$, treated with CaMKIl or buffer (control), was subjected to SDS polyacrylamide gel electrophoresis (SDS-PAGE) and transferred to membranes. These were then incubated overnight at $4^{\circ} \mathrm{C}$ with $2 \mathrm{~kg} \mathrm{ml^{-1 }}$ of MBP-tagged PBD or His-Plxl. Bound protein was detected using affinity-purified rabbit antibodies to $\mathrm{Plxl}$.

Xenopus extracts. Xenopus CSF egg extracts were prepared as described previously ${ }^{20}$. CSF release was induced by adding $1 \mathrm{mM} \mathrm{CaCl}$ to the extract. DNA and spindle morphology were examined as described previously ${ }^{21}$. CSFreleased extract was arrested at anaphase by the addition of non-degradable cyclin B ( $\Delta 90$ extract) as described ${ }^{14, i 5}$. The $\Delta 90$ extract was treated with $300 \mu . M$ EGTA and CaMKII was re-activated by the addition of $600 \mu \mathrm{M}$ calcium. Where indicated, MBP-PBD ${ }^{W T}$ or MBP-PBD ${ }^{\text {mut }}$ was added to the extract to a final concentration of $400 \mu \mathrm{g} \mathrm{ml}^{-1}$. For some experiments extract was treated with cycloheximide. The immunodepletion of Plxl was performed as described ${ }^{22}$.

Received 19 May; accepted 5 August 2005.

Published online 28 August 2005.

1. Tunquist, B. J. \& Maller, J. L. Under arrest: cytostatic factor (CSF)-mediated metaphase arrest in vertebrate eggs. Genes Dev. 17, 683-710 (2003).

2. Masui, Y.\& Markert, C. L. Cytoplasmic control of nuclear behaviour during meiotic maturation of frog oocytes. J. Exp. Zool. 177, 129-145 (1971).

3. King, R. W. et al. A 205 complex containing CDC27 and CDC15 catalyzes the mitosis-specific conjugation of ubiquitin to cyclin B. Cell 81, 279-288 (1995)

4. Sudakin, $V$. et al. The cyclosome, a large complex containing cyclin-selective ubiquitin ligase activity, targets cyclins for destruction at the end of mitosis. Mol. Biol. Cell 6, 185-197 (1995).

5. Salah, S. M. \& Nasmyth, K. Destruction of the securin Pdsip occurs at the onset of anaphase during both meiotic divisions in yeast. Chromosoma 109 , 27-34 (2000)

6. Busa, W. B. \& Nuccitelli, R. An elevated free cytosolic $\mathrm{Ca}^{2+4}$ wave follows fertilization in eggs of the frog, Xenopus laevis. J. Cell Biol. 100, 1325-1329 (1985).

7. Lorca, T. et al, Degradation of the proto-oncogene product $\mathrm{p} 39$ mos is not necessary for cyclin proteolysis and exit from meiotic metaphase: requirement for a Ca(2+)-calmodulin dependent event. EMBO J. 10, 2087-2093 (1991)

8. Lorca, $T$. et al. Calmodulin-dependent protein kinase II mediates inactivation of MPF and CSF upon fertilization of Xenopus eggs. Nature 366, 270-273 (1993).

9. Schmidt, A. et al. Xenopus polo-like kinase PlX1 regulates XErpl, a novel inhibitor of APC/C activity. Genes Dev. 19, 502-513 (2005).

10. Fuchs, S. Y., Spiegelman, V. S. \& Kumar, K. G. The many faces of beta-TrCP E3 ubiquitin igases: reflections in the magic mirror of cancer. Oncogene 23, $2028-2036$ (2004). 
11. Descombes, $P$. \& Nigg, E. A. The polo-like kinase $P|x|$ is required for $M$ phase exit and destruction of mitotic regulators in Xenopus egg extracts. EMBO J. 17 1328-1335 (1998)

12. Elia, A. E., Cantley, L. C. \& Yaffe, M. B. Proteomic screen finds pSer/pThrbinding domain localizing PJkT to mitotic substrates. Science 299, 1228-1231 (2003).

13. Elia, A. E. et al. The molecular basis for phosphodependent substrate targeting and regulation of Plks by the Polo-box domain. Cell 115, 83-95 (2003).

14 Holloway, S. L. Glotzer, M. King, R. W. \& Murray, A. W. Anaphase is initiated by proteolysis rather than by the inactivation of maturation-promoting factor. Cell 73, 13393-13402 (1993).

15. Stemmann, O., Zou, H., Gerber, S. A., Gygi, S. P. \& Kirschner, M. W. Dual inhibition of sister chromatid separation at metaphase. Cell 107, 715-726 (2001)

16. Colbran, R. J., Fong, Y. L., Schworer, C. M. \& Soderling, T. R. Regulatory interactions of the calmodulin-binding, inhibitory, and autophosphorylation domains of $\mathrm{Ca}^{2+} /$ calmodulin-dependent protein kinase II. J. Biol. Chem. 263 18145-18151 (1988).

17. Zachariae, W. Emil and Erpl: who can stop these eggs? Dev. Cell 8, 301-303 (2005).

18. Ohsumi, K., Koyanagi, A., Yamamoto, T. M., Gotoh, T. \& Kishimoto, T. Emi1mediated $M$-phase arrest in Xenopus eggs is distinct from cytostatic factor arrest. Proc. Natl Acad. Sci. USA 101, 12531-12536 (2004).
19. Tung, J. J. et al. A role for the anaphase-promoting complex inhibitor Emi2/ XErpl, a homolog of early mitotic inhibitor 1 , in cytostatic factor arrest of Xenopus eggs. Proc. Natl Acad. Sci. USA 102, 4318-4323 (2005)

20. Murray, A. W. Cell cycle extracts. Methods Cell Biol. 36, 581-605 (1991).

21. Desai, A., Murray, A., Mitchison, T. J. \& Walczak, C. E. The use of Xenopus egg extracts to study mitotic spindle assembly and function in vitro. Methods Cell Biol. 61, 385-412 (1999).

22. Funabiki, H \& Murray, A. W. The Xenopus chromokinesin Xkid is essential for metaphase chromosome alignment and must be degraded to allow anaphase chromosome movement. Cell 102, 411-424 (2000).

Acknowledgements $W$ e thank $O$. Stemmann and $F$. Barr for reagents and discussions during this work. We are grateful to 1 . Gorr and D. Boos for advice on $\Delta 90$ experiments, S. Hümmer for continuous support and R. Neef for assistance with the far-western analyses.

Author Information Reprints and permissions information is available at npg.nature.com/reprintsandpermissions. The authors declare no competing financial interests. Correspondence and requests for materials should be addressed to T.U.M. (mayer@biochem.mpg.de). 